\title{
Experimental observation of invariance of spectral degree of coherence with change in bandwidth of light
}

\author{
Bhaskar Kanseri* and Hem Chandra Kandpal \\ Optical Radiation Standards, National Physical Laboratory (Council of Scientific and Industrial Research), \\ New Delhi 110012, India \\ *Corresponding author: kanserib@mail.nplindia.ernet.in
}

Received August 28, 2009; accepted November 16, 2009; posted December 10, 2009 (Doc. ID 116391); published December 24, 2009

\begin{abstract}
An experimental study is conducted to show the effect of the change in bandwidth of light on the spectral degree of coherence at a pair of points in the cross section of a beam. For this purpose a polychromatic source and a monochromator with variable entrance and exit slits were used to produce a variable bandwidth source. The classic Young's interferometer was used to produce an interference pattern. The spectral measurements of the visibility of the interference fringes show that the spectral degree of coherence remains unaffected by the change in the frequency passband of the light. (C) 2009 Optical Society of America

OCIS codes: $030.0030,030.1640,120.0120$.
\end{abstract}

In the past several decades, partially coherent light has been investigated theoretically and experimentally with great interest [1]. The central quantities in these studies were the complex degree of coherence both in space-time [2] and in space-frequency domains [3]. The effects of spatial correlations (source correlations) on the spectral properties of light were studied in detail, and many applications were suggested [4]. In the space-frequency domain, the quantification of coherence is done by the degree of spectral coherence. One might expect that the degree of spectral coherence would increase when identical filters centered at the same frequency, but narrow bandpass, are put before both slits of the interferometer. The theoretical study of this effect made by Wolf [5] in 1983 concludes otherwise. In 2003, Basano et al. [6,7] observed this effect experimentally. One of the important results of these papers $[5,6]$ is that the degree of spectral coherence is unchanged by filtering. In the recent past, a study showing the relation between space-time and space-frequency complex degree of coherence was reported [8]; however, a direct observation of the invariance of spectral visibility is still lacking.

In this Letter it is shown qualitatively and quantitatively that the spectral degree of coherence does not change with the change in bandwidth of the source. Instead of using a tunable interference filter as a secondary source, we used a scheme different from that adopted by Basano et al. [6]. A variable bandwidth source was constructed by using a variable slit monochromator placed in line with a polychromatic source. The bandwidth of the secondary source was altered by simultaneously changing the entrance and the exit slit of the monochromator by the same amount. A double-slit after the synthesized secondary source was used to obtain the interference fringes on an observation plane. The spectral visibility of the fringes with respect to the change in bandwidth of light was measured and was found to be nearly constant.
In the Young's interference experiment, the absolute value of the spectral degree of coherence $\left|\mu\left(\mathbf{r}_{1}, \mathbf{r}_{2}, \omega\right)\right|$, also known as spectral visibility $\nu(\mathbf{r})$, is a direct measure of the contrast of the interference fringes formed by using quasi-monochromatic light. When the spectral densities at the observation point due to separate slits are approximately the same, one can readily find [1]

$$
\nu(\mathbf{r})=\left|\mu\left(\mathbf{r}_{1}, \mathbf{r}_{2}, \omega\right)\right|=\frac{S_{\max }-S_{\min }}{S_{\max }+S_{\min }},
$$

where $S_{\max }$ and $S_{\min }$ are, respectively, the maximum and the minimum values of spectral density around the observation point. The cross-spectral density of light is given by [1]

$$
W\left(\mathbf{r}_{1}, \mathbf{r}_{2}, \omega\right)=\left\langle U^{*}\left(\mathbf{r}_{1}, \omega\right) U\left(\mathbf{r}_{2}, \omega\right)\right\rangle,
$$

where $U\left(\mathbf{r}_{i}, \omega\right) \exp (-i \omega t)$ denotes an ensemble of the monochromatic oscillations, and the angular brackets show the ensemble average.

It was shown by Wolf [5] that when two identical filters having amplitude transmission function $T(\omega)$ are put after the double-slit, covering both slits, then the light emerging from the filters is given by the ensemble $T(\omega) U\left(\mathbf{r}_{i}, \omega\right) \exp (-i \omega t)$. From Eq. (2), the cross-spectral density of the filtered light will be given by [5]

$$
W^{+}\left(\mathbf{r}_{1}, \mathbf{r}_{2}, \omega\right)=|T(\omega)|^{2} W\left(\mathbf{r}_{1}, \mathbf{r}_{2}, \omega\right) .
$$

If we assume that $W\left(\mathbf{r}_{1}, \mathbf{r}_{2}, \omega\right)$ is a continuous function of frequency $\omega$ and that $\Delta \omega$ is so small that $W\left(\mathbf{r}_{1}, \mathbf{r}_{2}, \omega\right)$ does not appreciably change across the effective passband $\omega_{0}-\frac{1}{2} \Delta \omega \ll \omega \ll \omega_{0}+\frac{1}{2} \Delta \omega$ of the filters, then we can replace $W\left(\mathbf{r}_{1}, \mathbf{r}_{2}, \omega\right)$ by $W^{+}\left(\mathbf{r}_{1}, \mathbf{r}_{2}, \omega\right)$ in Eq. (2). The complex degree of spectral coherence for the unfiltered light of central frequency $\omega_{0}$ is given by $[1,5]$ 


$$
\mu\left(\mathbf{r}_{1}, \mathbf{r}_{2}, \omega_{0}\right)=\frac{W\left(\mathbf{r}_{1}, \mathbf{r}_{2}, \omega_{0}\right)}{\left[W\left(\mathbf{r}_{1}, \mathbf{r}_{1}, \omega_{0}\right)\right]^{1 / 2}\left[W\left(\mathbf{r}_{2}, \mathbf{r}_{2}, \omega_{0}\right)\right]^{1 / 2}} .
$$

In a similar way, the complex degree of spectral coherence at frequency $\omega_{0}$ of the filtered light at the two points is written as [5]

$$
\mu^{+}\left(\mathbf{r}_{1}, \mathbf{r}_{2}, \omega_{0}\right)=\frac{W^{+}\left(\mathbf{r}_{1}, \mathbf{r}_{2}, \omega_{0}\right)}{\left[W^{+}\left(\mathbf{r}_{1}, \mathbf{r}_{1}, \omega_{0}\right)\right]^{1 / 2}\left[W^{+}\left(\mathbf{r}_{2}, \mathbf{r}_{2}, \omega_{0}\right)\right]^{1 / 2}} .
$$

Using Eq. (3) with Eqs. (4) and (5), we get [5]

$$
\mu^{+}\left(\mathbf{r}_{1}, \mathbf{r}_{2}, \omega_{0}\right)=\mu\left(\mathbf{r}_{1}, \mathbf{r}_{2}, \omega_{0}\right) ;
$$

i.e., the complex degree of spectral coherence remains unchanged by filtering. From Eq. (6), we can readily see that the absolute values of these quantities, which can be calculated experimentally by using Eq. (1), will also be equal.

The experimental arrangement is shown in Fig. 1. A tungsten-halogen lamp S (Mazda, color temperature $3200 \mathrm{~K}$ ), operated at $700 \mathrm{~W}$ by using a regulated dc power supply (Heinzinger, stability 1 part in $10^{4}$ ) was utilized as a polychromatic continuous spectrum source. The outer glass jacket of the lamp was diffused (D) to obtain uniform illumination. The light coming from the source was passed through microprocessor (MP)-controlled monochromator M (CVI, Digikrom) having variable entrance and exit slits (1 and 2 in Fig. 1). The beam emerging from the exit slit of the monochromator was made incident on singleslit SS (slit width $0.5 \mathrm{~mm}$ ) placed $40 \mathrm{~cm}$ from the exit slit. The beam emerging from SS illuminated a double-slit plane DS having rectangular slits with slit width $0.15 \mathrm{~mm}$ and slit separation $0.35 \mathrm{~mm}$ and placed $80 \mathrm{~cm}$ from SS. Interference fringes were obtained at the observation plane $\mathrm{R}$ at a distance of $40 \mathrm{~cm}$ from DS. The spectral measurements were carried out by using fiber (F)-coupled spectrometer SM (Photon Control, SPM002) interfaced with a personal computer (DP).

The central wavelength of the output beam was selected at $555 \mathrm{~nm}$. This wavelength was chosen because of its maximum sensitivity for the human eye, providing convenience in aligning the optics. Both slits of the monochromator were opened by the same width so that the output beam spectrum could remain centered at the desired wavelength [9]. Changing the width of both slits by a known amount changed the bandwidth of the spectra. Figure 2(a) shows the graphical representation of the light spectra measured near the exit slit for different slit widths. The behavior of the bandwidth of light with the monochromator slit width, when SS was intro-

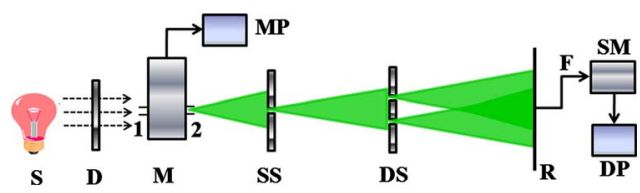

Fig. 1. (Color online) Schematic of the experimental setup. Abbreviations are defined in the text.

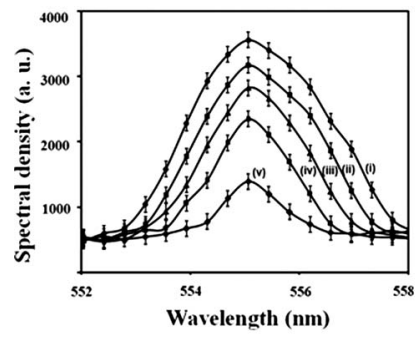

(a)

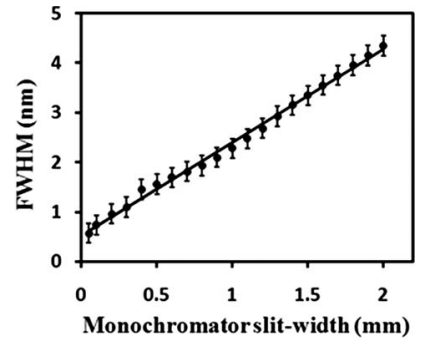

(b)
Fig. 2. (a) Plot of spectral density versus slit width of the monochromator. Curves are shown for slit widths (i) 2, (ii) 1.5 , (iii) 1 , (iv) 0.5 , and (v) $0.1 \mathrm{~mm}$. Dots show the data points connected by the solid curve. (b) FWHM of the spectra (after inserting SS) plotted with respect to the slit width. The trend line shows a linear fit. The error bars show the uncertainty in the mean value calculated at a $95 \%$ confidence level.

duced, is shown in Fig. 2(b). We observe that, in both cases, by increasing the slit opening the bandwidth of the beam could be increased, and the inverse. However, for smaller slit widths, the transmitted light intensity is also reduced, which demands sensitive detectors for measurement. The use of the monochromator provided operational flexibility.

Partial coherence develops when radiation propagates, even in free space [1]. In the far zone, the diameter of the region outside which there is complete incoherence is given by the van Cittert-Zernike theorem [1] and is proportional to $\bar{\lambda} L / \rho$ [10] for a rectangular slit, where $\bar{\lambda}$ is the central wavelength, $\rho$ is the slit width, and $L$ is the distance of the observation plane from the single slit. Thus when SS is not introduced, the exit slit width of the monochromator plays the role of deterministic slit width for the developed coherence region at the double-slit plane. This effect is evident from the photograph of the double-slit diffraction patterns for different slit widths of the monochromator, as shown in Fig. 3(a). We see that for a wider slit opening, the coherence developed at plane DS is lower, resulting in suppression of the interference effect. When the slit spacing is decreased, according to van Cittert-Zernike theorem, the coherence developed at plane DS increases, and the interference effects start to dominate. As shown in

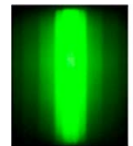

(i)

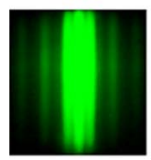

(i)

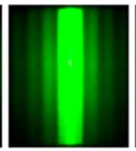

(ii)

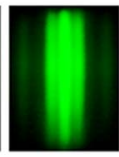

(ii)

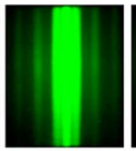

(iii)

(a)

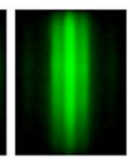

(iii)

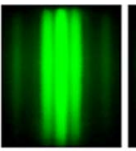

(iv)

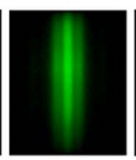

(iv) (v)
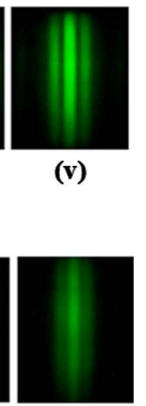

(v) (b)

Fig. 3. (Color online) Two-slit diffraction patterns for different slit widths (a) without SS and (b) when SS is introduced (Fig. 1). In both the cases, the slit widths are (i) 2, (ii) 1.5 , (iii) 1 , (iv) 0.5 , and (v) $0.1 \mathrm{~mm}$. 
part (v) of Fig. 3(a), for a very small slit separation, only the interference effects remain. This feature confirms the role of the van Cittert-Zernike theorem, which establishes an inverse relation between the slit width and the developed degree of coherence at any observation plane. To observe the behavior of the degree of spectral coherence with the bandwidth, the coherence at the double-slit should not be influenced by the other factors, namely, slit width etc. However, this is not the case in this study.

In this experiment, to achieve this feat, we modified the setup by introducing SS having a constant slit width between M and DS (Fig. 1). This additional slit isolates the effect of slit width variation on the spatial coherence region developed at plane DS. Thus the spatial coherence region does not effectively change throughout the experiment and remains invariant with the geometrical conditions of the monochromator slits. However, introduction of SS does not appreciably affect the bandwidth of the emerging light, and the dependence of the bandwidth on the slit width remains linear (see Fig. 2). Thus the use of a monochromator with the single-slit plays the role of the tunable filter mentioned in [5].

The photographs of interference fringes obtained for different bandwidths of light are shown in Fig. 3(b). A careful look at the interference fringes shows that there is a very small change in the visibility of the fringes with the change in bandwidth, though the intensity of the fringes reduces rapidly with the decrease in bandwidth. The observed small increment in fringe visibility is due to the fact that the complex degree of coherence (time domain) undergoes a slight enhancement with the decrease in bandwidth of light [5]. It is worth recalling that the effective bandwidth of the light emerging from the monochromator, i.e., $3 \times 10^{12} \mathrm{~Hz}$ was so narrow $\left(3 \times 10^{12} \mathrm{~Hz} \leqslant 5 \times 10^{14} \mathrm{~Hz}\right.$, peak frequency) that both the modulus and the phase of $W\left(\mathbf{r}_{1}, \mathbf{r}_{2}, \omega\right)$ and the spectral densities $S_{1}(\mathbf{r})$ and $S_{2}(\mathbf{r})$ for the pair of points could be assumed to be substantially constant.

To validate our findings quantitatively, the spectral measurements were made with the spectrometer by tracing the fiber tip horizontally across the interference fringes. The spectral densities due to individual slits were measured at the axial point and were found to be approximately the same, i.e., $S_{1}(\mathbf{r})$ $\approx S_{2}(\mathbf{r})$. The spectral visibility of the fringes was determined by using Eq. (1). A plot of absolute value of degree of spectral coherence with the change in bandwidth of light is shown in Fig. 4. The slit width scale was changed into the bandwidth scale using the graphical equivalence shown in Fig. 2(b). We observe that the degree of spectral coherence remains nearly constant throughout the bandwidth range within the experimental uncertainty calculated at $95 \%$ confi-

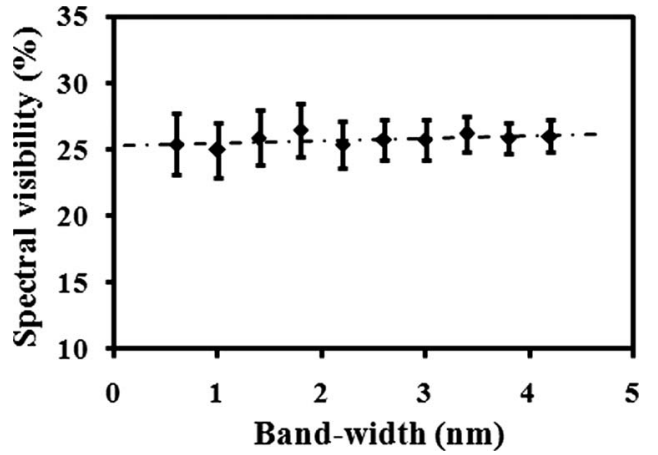

Fig. 4. Change in absolute value of the degree of spectral coherence (spectral visibility) with the bandwidth of light. The dashed line is a trend line showing a linear fit.

dence level. The higher variance in the repeat measurements for low bandwidth regime may be due to the limited sensitivity of the spectrometer at low intensity levels.

In a nutshell, the behavior of the spectral degree of coherence is studied experimentally with the change in bandwidth of the light. It is found that, like the theoretical outcome, this quantity remains invariant with these bandwidth variations in the source spectra. This experimental finding not only verifies the invariance of degree of spectral coherence with filtering of light but also paves the way for future work on finding the relation between frequency-domain and time-domain coherence functions.

We express our thanks to the Director, National Physical Laboratory, New Delhi for permission to publish this paper. B. Kanseri thanks the Council of Scientific and Industrial Research (CSIR), India for the financial support of a Senior Research Fellowship.

\section{References}

1. L. Mandel and E. Wolf, Optical Coherence and Quantum Optics (Cambridge Univ. Press, 1995).

2. M. Born and E. Wolf, Principles of Optics, 7th ed. (Cambridge Univ. Press, 1999), Chap. 4.

3. L. Mandel and E. Wolf, J. Opt. Soc. Am. 66, 529 (1976).

4. E. Wolf and D. F. V. James, Rep. Prog. Phys. 59, 771 (1996).

5. E. Wolf, Opt. Lett. 8, 250 (1983).

6. L. Basano, P. Ottonello, G. Rottigni, and M. Vicari, Appl. Opt. 42, 6239 (2003).

7. E. Wolf, Introduction to Theory of Coherence and Polarization of Light (Cambridge Univ. Press, 2007), p. 76.

8. A. T. Friberg and E. Wolf, Opt. Lett. 20, 623 (1995).

9. C. A. Parker, Photoluminescence of Solutions (Elsevier, 1968), Chap. 3.

10. A. S. Marathay, Elements of Optical Coherence Theory (Wiley, 1982), p. 111. 